\title{
Simplified method using kidney / ureter / bladder x-ray to determine the appropriate length of ureteral stents
}

\author{
Makoto Taguchi ${ }^{1}$, Kenji Yoshida ${ }^{1}$, Motohiko Sugi ${ }^{1}$, Hidefumi Kinoshita ${ }^{1}$, Tadashi Matsuda ${ }^{1}$ \\ ${ }^{1}$ Department of Urology and Andrology, Kansai Medical University, Osaka, Japan
}

\section{ABSTRACT}

Purpose: To investigate a method to determine the appropriate length of ureteral stents, given that the stent length may lead to exacerbation of urinary symptoms if the stent crosses the bladder midline.

Materials and Methods: We retrospectively reviewed the position of the distal curl of the ureteral stent using kidney/ureter/bladder (KUB) radiographs after ureteroscopic lithotripsy in 165 patients who underwent placement of 24- or 26-cm ureteral stents. According to the KUB findings, we categorized the position of the distal curl of the ureteral stent into two groups. In Group 1, the stents did not cross the midline (appropriate length); in Group 2, the stents crossed the midline (inappropriate length). We assessed several patient parameters (sex, height, body mass index, and stone side) and the index of ureteral length using KUB radiographs ("C-P") and computed tomography (CT, "PV"). Multivariate analysis was performed to identify the most significant factors affecting the position of ureteral stents. We also calculated the cutoff points of the receiver operating characteristic (ROC) curve of C-P and P-V for the position of ureteral stents. Results: The multivariate analysis showed that C-P was the most significant factor affecting the position of ureteral stents $(\mathrm{p}<0.001)$ in patients with $24-$ and $26-\mathrm{cm}$ ureteral stents. Comparison of the ROC curves of C-P and P-V showed that C-P was superior to $\mathrm{P}-\mathrm{V}(\mathrm{p}<0.01)$ in patients with $24-$ and $26-\mathrm{cm}$ stents.

Conclusion: The use of KUB radiographs was effective and simple in determining the appropriate length of ureteral stents.

\section{ARTICLE INFO}

\section{Keywords:}

Kidney; Ureter; Urinary Bladder

Int Braz J Urol. 2018; 44: 1224-33

Submitted for publication:

November 22, 2017

Accepted after revision:

July 09, 2018

Published as Ahead of Print:

August 03, 2018

\section{INTRODUCTION}

Since Zimskind et al. (1) introduced ureteral stents in 1967, such stents have become widely used for the maintenance of renal function, pain relief, and the treatment of urinary tract infections. However, many complications of ureteral stenting have been reported, such as incomplete emptying, bladder pain, frequency, hematuria, and migration. In one study, ureteral stenting reportedly decreased the urination-related quality of life
(QOL) in $80 \%$ of patients who underwent ureteral stenting (2). Several factors have been investigated for their effects on ureteral stent - related symptoms, including stent length, $(3,4)$ diameter, (5-7) material, (7) softness, (8) position, (9) and loop completeness (3). Among these factors, determination of the most appropriate ureteral stent length assumes importance in reducing stent-related complications. Some studies have revealed that placement of overly long ureteral stents that cross the bladder midline can lead to worsening of 
urinary symptoms $(3,10,11)$. Therefore, we consider the position of the ureteral stent to be an important factor in stent-related surgery. In the present study, we evaluated a method to determine the appropriate ureteral stent length and ensure that the stent does not cross the bladder midline.

The optimal method for determining the appropriate ureteral stent length remains unclear. In previous reports, the appropriate ureteral stent length for each patient was calculated by three different methods. The first is direct measurement of the ureter itself using a guide wire or ureteral catheter (12-16). The second involves measurement of the distance from the pelviureteric junction (PUJ) to the vesicoureteric junction (VUJ) by either retrograde or intravenous pyelography (1619). The third method provides an estimation of the appropriate stent length using a formula based on the patient's height. The patient's height is reportedly a more reliable guide for obtaining an appropriate ureteral stent length than direct ureteral measurement using a guide wire and ureteral catheter $(12,13,15,16,18,19)$. However, there is no standard and simplified method for determining the appropriate ureteral stent length that prevents a decline in urination-related QOL. Moreover, in some hospitals, assorted lengths of ureteral stents are not stocked, and preoperative prediction of ureteral stent lengths is often needed. In this study, therefore, we measured the distance between two points on a kidney / ureter / bladder (KUB) radiograph using retrospective data and evaluated predictors to place ureteral stents (of lengths 24 and $26 \mathrm{~cm}$ ) so as not to cross the bladder midline. We have developed a predictive and simplified method for determination of the appropriate length of ureteral stents using KUB radiographs with the aim of reducing urination-related symptoms and concomitant QOL.

\section{MATERIALS AND METHODS}

\section{Study population}

This study was approved by our institutional review board (authorization number: H160741). From January 2013 to December 2015, 168 of 204 patients who underwent ureteroscopic lithotripsy and ureteral stent insertion were enrolled. At the end of the procedure, each patient underwent placement of a ureteral stent (Inlay Optima; C.R. Bard Inc., NJ, USA or Polaris Ultra; Boston Scientific, MA, USA). The diameter of all ureteral stents was $6 \mathrm{~F}$ and the length was 24 or 26 $\mathrm{cm}$ according to the surgeon's discretion. All the stents were placed with full curls in the bladder and kidney.

The exclusion criteria were severe body deformity or disability, a duplicate collecting system, renal ectopia, reimplantation using a psoas hitch, vaginal vault eversion beyond the introitus, and a proximal loop in the upper calyx (Figure-1).

\section{Patient parameters}

We assessed several parameters to evaluate the correlation between these characteristics and the appropriate ureteral stent length. Patient demographics including age, gender, height, body weight, body mass index (BMI), and stone side were reviewed. We calculated the length as the index to choose appropriate ureteral stent length for not crossing the bladder midline using KUB radiographs and computed tomography (CT).

\section{Measurement of index using KUB radiographs}

We measured the index using preoperative KUB films. KUB filming conditions were standardized at maximum inspiration in the supine position, and imaging was performed at $70 \mathrm{kV}$ and $132 \mathrm{~mA}$. The index used in this study was the length from the central renal point to the midpoint of the superior margin of the pubis (C-P), measured on KUB films (Figures 2A and B). The central renal point was defined as the midpoint of the distance from the extremitas superior renis to the extremitas inferior renis.

\section{Measurement of index using CT}

All patients were scanned with a 64-slice CT scanner $(120 \mathrm{kV}, 200 \mathrm{~mA}$, and 5-mm slice thickness). We also calculated the length from the PUJ to the VUJ (P-V) using CT and the Pythagorean theorem and compared this method with the above-described method to determine which more effectively predicts the appropriate ureteral stent length. The CT index was calculated using Cares- 
Figure 1 - Flowchart of inclusion process.

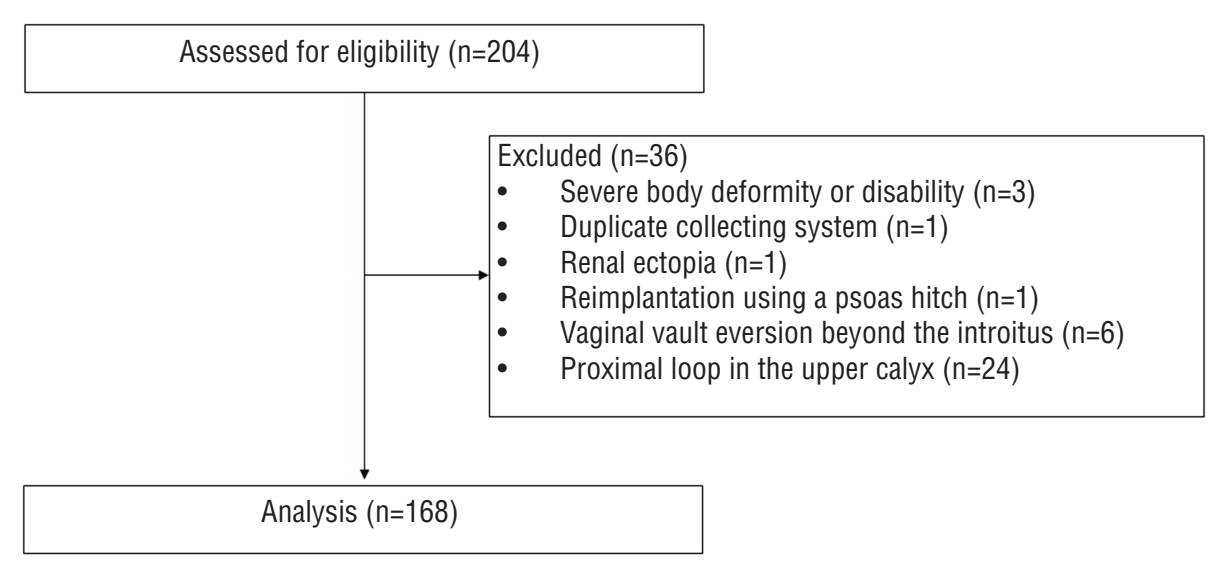

Figure 1 Flowchart of inclusion process.

tream Vue PACS (Carestream Health, Rochester, NY, USA), and all CT images were reviewed by a single urologist (M.T.) with 5 years of experience as an urologist. First, in the CT slice showing the PUJ, we marked the point of the PUJ (Figures $2 \mathrm{C}$ and $\mathrm{D}$, star). Next, in the CT slice showing the VUJ, we marked the corresponding point for the PUJ slice (Figures 2E and F, star) and measured the distance from the VUJ (Figures 2E and F, square) to the marked point (Figures 2E and F, star) in the CT slice showing the VUJ. We defined this length as the short side of a right-angled triangle (Figures $2 \mathrm{E}$ and $\mathrm{F}$; from star to square). We then defined the length of the long side of a right-angled triangle, calculated by the total number of slices between the slice showing the PUJ (Figure-2G, star) and the VUJ (Figure-2G, square). All slices were 5 $\mathrm{mm}$ thick (Figure-2G). Finally, we calculated the length of $\mathrm{P}-\mathrm{V}$ using the Pythagorean theorem ([P $-\mathrm{V}] 2=$ [short side $] 2+$ [long side $] 2$ )

\section{Definition of appropriate ureteral stent length}

We routinely obtained KUB films to confirm the presence of residual stones on postopera- tive day 1 . We retrospectively reviewed the position of the ureteral stents using these KUB films. All KUB films were reviewed by a single urologist (M.T.). We categorized the patients into two groups according to the position of the distal curl of the ureteral stent on the KUB films using the technique described by Giannarini et al. (11) In Group 1 , the stent did not cross the midline (appropriate length of ureteral stent, Figure-3A); in Group 2, the stent crossed the midline (inappropriate length of ureteral stent, Figure-3B).

\section{Statistical analysis}

In each group, both of which included patients with $24-$ and $26-\mathrm{cm}$ ureteral stents, we evaluated the correlation between the position of the ureteral stents and various patient parameters: age, gender, height, body weight, BMI, stone side, C-P, and P-V. Univariate analysis was performed using either the Mann-Whitney U-test or the $\chi 2$ test to evaluate the correlation between the position of the ureteral stents and patient parameters. Multivariate analysis was performed 
Figure $2(A-G)$ - Measuring the length of C-P and P-V.

A

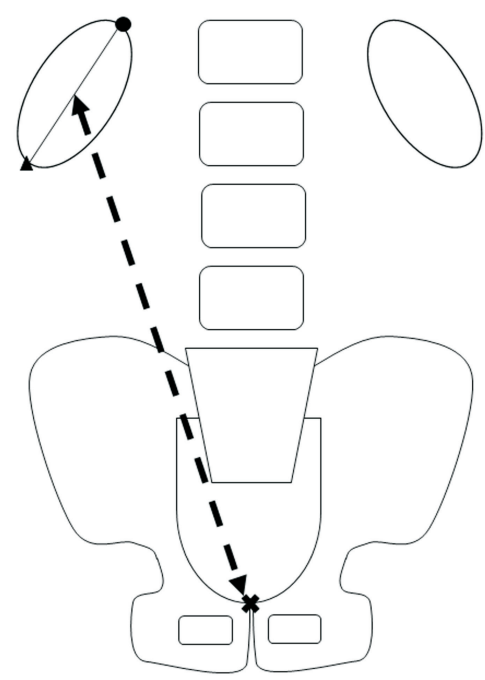

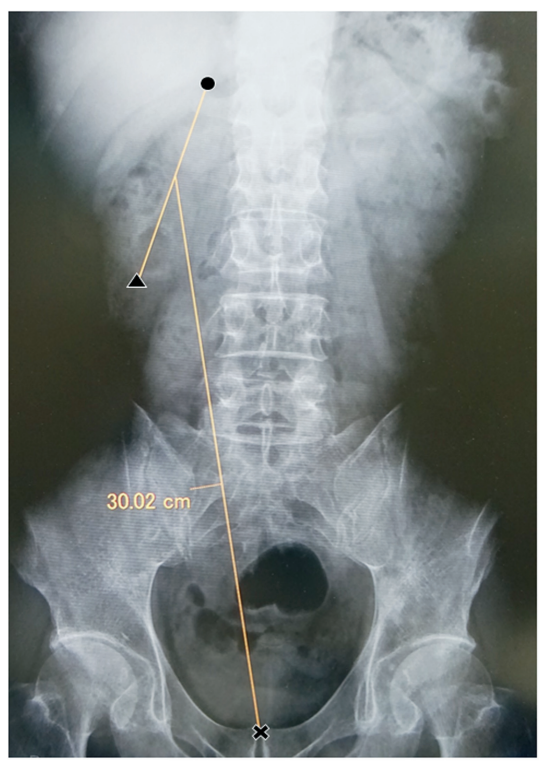

$\leftarrow \rightarrow$ : The length of C-P

C-P is the length from the central renal point to the midpoint of the superior margin

of the pubis. The central renal point is defined as the midpoint of the distance from

the superior to inferior pole of the kidney.

D

G
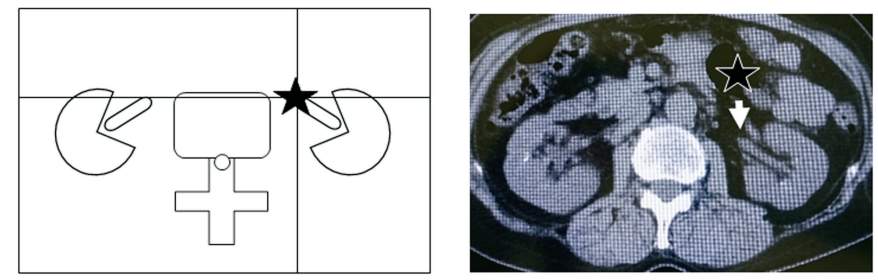

E

$\mathbf{F}$
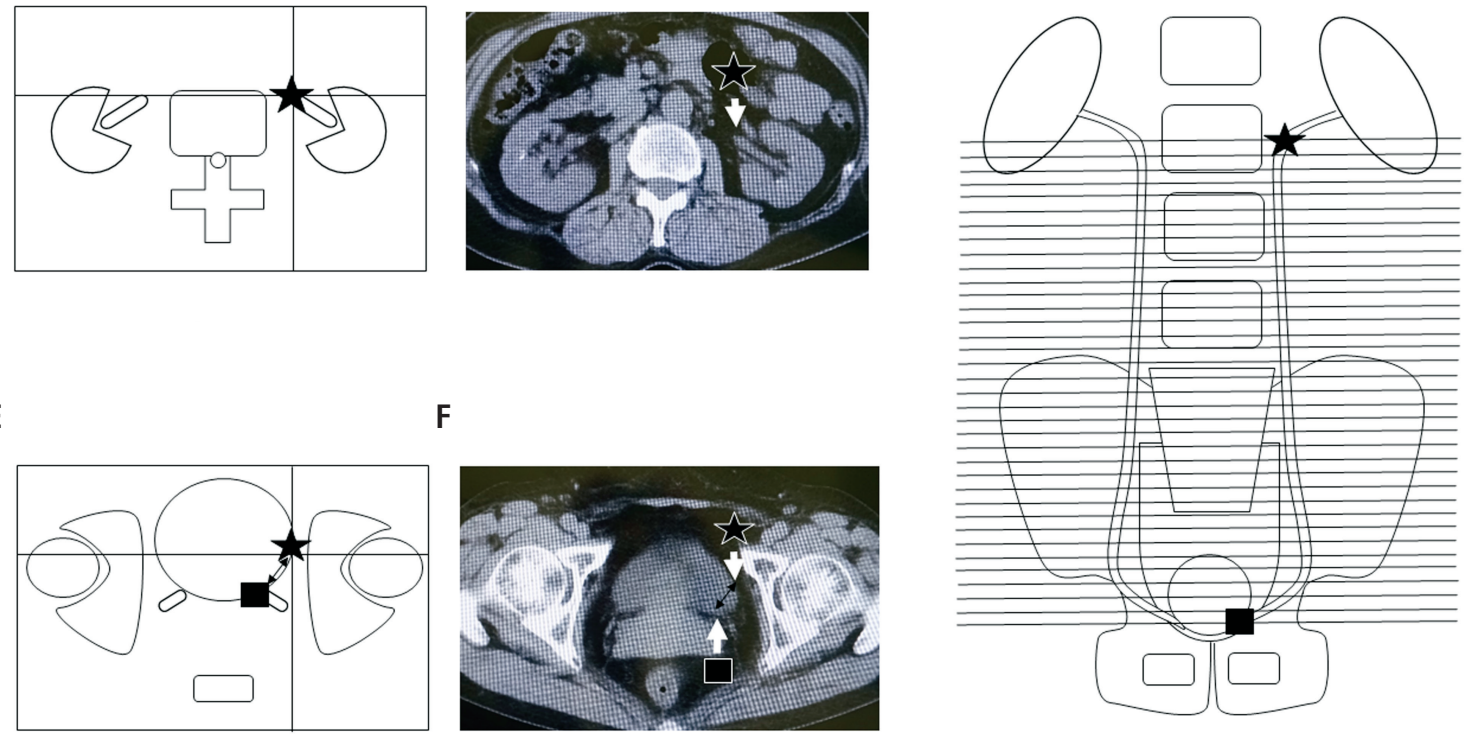

C-G: Measurement of the length of P-V using Pythagorean theorem

G: Measurement of the long side of a right-angled triangle.

$\mathrm{C}-\mathrm{F}$ : Measurement of the short side of a right-angled triangle

$\star$ : The point of the pelviureteric junction (PUJ)

Length $=$ total number of slices between the slice showing the PUJ and the VUJ $\mathrm{x}$ slice

thickness.

-: Computed tomography (CT) slice. All of the slices were $5 \mathrm{~mm}$ thick.

$\leftrightarrow$ : The length of the short side of a right -angled triangle

A, B - C-P is the length from the central renal point to the midpoint of superior margin of the pubis. Central renal point is defined as the midpoint of distance from extremitas superior renis to extremitas inferior renis. / C, D, E, F, G - Measuring the length of P-V using Pythagorean theorem. / C, D, E, F - Measuring the short side of a right-angled triangle / $\mathbf{G}$ - Measuring the long side of a right-angled triangle. / It length $=$ the total number of slices between the slice showing the PUJ and the VUJ $\times$ slice thickness. 
Figure $3(A, B)$ - Classification of the intravesical ureteral stent position. (A) Not crossing midline (Group 1). (B) Crossing midline (Group 2).
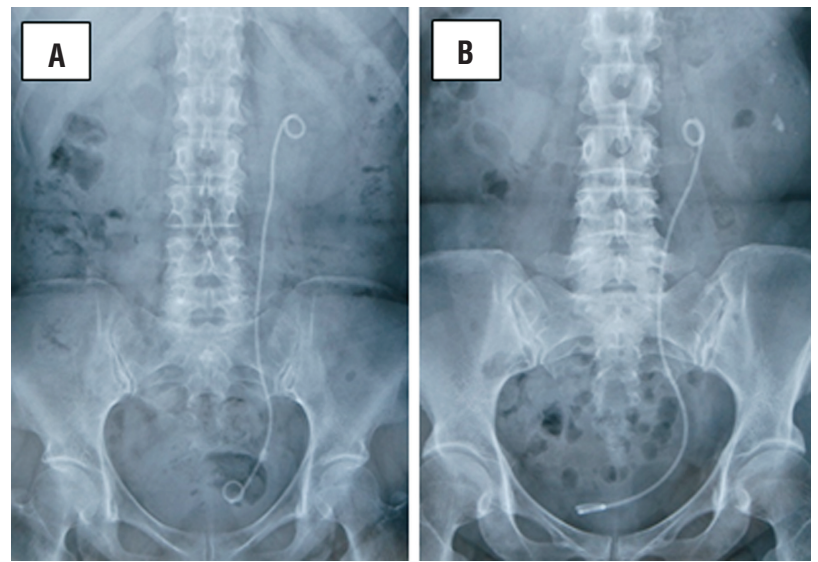

using a logistic regression model to identify the most significant factors affecting the position of the ureteral stents. Furthermore, we calculated the cutoff points of the receiver operating characteristic (ROC) curve, area under the ROC curve (AUROC), and 95\% confidence interval (CI) of the $\mathrm{C}-\mathrm{P}$ and $\mathrm{P}-\mathrm{V}$ for the position of the ureteral stents. Data were analyzed using the IBM SPSS Statistics V21.0 software package.

\section{RESULTS}

Table-1 shows the patients' demographic data. In Group 1, 46 (59.0\%) and 46 (51.1\%) patients had 24- and 26-cm indwelling stents, respectively. In Group 2, 32 (41.0\%) and 44 (48.9\%) patients had 24- and 26-cm indwelling stents, respectively.

Table-2 shows the results of the univariate and multivariate analyses performed to evaluate the correlation between the position of the ureteral stents and patient parameters. Comparison of Groups 1 and 2 using univariate analysis revealed no significant differences in age, gender,

Table 1 - The demographic data of patients with indwelling $24-\mathrm{cm}$ and $26-\mathrm{cm}$ ureteral stents.

\begin{tabular}{lcc}
\hline & $24 \mathrm{~cm}$ ureteral stents & $26 \mathrm{~cm}$ ureteral stents \\
\cline { 2 - 3 } & \multicolumn{2}{c}{$\mathrm{n}(\%)$ or median (range) } \\
\hline Patients & 78 & 90 \\
Age (years) & $62.5(92-33)$ & $55(26-84)$ \\
Gender & $29(37.2)$ & $85(94.4)$ \\
$\quad$ male & $49(62.8)$ & $5(5.6)$ \\
$\quad$ female & $1.57(1.39-1.81)$ & $1.65(1.45-1.85)$ \\
Height (m) & $59.2(30.2-118.2)$ & $63.3(39-108.6)$ \\
Body weight (kg) & $24.2(15.4-35.2)$ & $23.7(17.8-36.1)$ \\
BMl (kg/m $\left.{ }^{2}\right)$ & & $58(64.4)$ \\
Stone side & $52(66.7)$ & $32(35.6)$ \\
$\quad$ left & $26(33.3)$ & \\
$\quad$ right & & $44(48.9)$ \\
Ureteral stent position & $32(41.0)$ & $46(51.1)$ \\
$\quad$ crossing midline & $46(59.0)$ & \\
not crossing midline & &
\end{tabular}

$\mathbf{B M I}=$ body mass index. 
height, body weight, BMI, or stone side in either the 24- or 26-cm group. However, C-P and P-V were significantly longer in Group 1 (not crossing midline) than Group 2 (crossing midline) in both the $24-$ and $26-\mathrm{cm}$ groups $(\mathrm{p}<0.001)$.
According to our multivariate analysis, C-P was the most significant factor affecting the position of the ureteral stents in both the 24- and $26-\mathrm{cm}$ groups ( $\mathrm{p}<0.001$ for both) (Tables $2 \mathrm{~A}$ and $\mathrm{B}$, respectively).

Table 2A - Multivariate analysis of patients with $24 \mathrm{~cm}$ ureteral stents.

\begin{tabular}{|c|c|c|c|c|c|c|}
\hline & \multirow{2}{*}{$\begin{array}{c}\text { Group } 1 \\
\text { (Not crossing } \\
\text { midline) }\end{array}$} & \multirow{2}{*}{$\begin{array}{c}\text { Group } 2 \\
\text { (Crossing midline) }\end{array}$} & \multirow{2}{*}{$\frac{\text { Univariate analysis }^{\mathrm{a}}}{\mathrm{p} \text {-value }}$} & \multicolumn{3}{|c|}{ Multivariate analysis $^{b}$} \\
\hline & & & & $p$-value & $\mathrm{OR}$ & $95 \% \mathrm{Cl}$ \\
\hline \multicolumn{7}{|l|}{ Gender } \\
\hline male & $17(37.0)$ & $12(37.5)$ & 0.98 & & & \\
\hline female & $29(63.0)$ & $20(62.5)$ & & & & \\
\hline Height (m) & $1.59(1.41-1.81)$ & $1.57(1.39-1.78)$ & 0.69 & & & \\
\hline BMI $\left(\mathrm{kg} / \mathrm{m}^{2}\right)$ & $24.3(17.1-32.0)$ & $23.8(15.4-35.2)$ & 0.99 & & & \\
\hline \multicolumn{7}{|l|}{ Stone side } \\
\hline left & $31(67.4)$ & $21(65.6)$ & 0.92 & & & \\
\hline right & $15(32.6)$ & $11(34.4)$ & & & & \\
\hline C-P & $28.5(25.5-33.9)$ & $26.1(19.9-28.8)$ & $<0.001$ & $<0.001$ & 7.445 & 2.689-20.612 \\
\hline$P-V$ & $20.2(16.7-25.3)$ & $19.1(14.7-21.2)$ & $<0.001$ & 0.331 & 0.966 & $0.901-1.036$ \\
\hline
\end{tabular}

${ }^{\text {a }}$ Mann-Whitney U-test; ${ }^{\mathbf{b}}$ Logistic regression analysis; $\mathbf{O R}=$ odds ratio; $\mathbf{C l}=$ confidence interval

Table 2B - Multivariate analysis of patients with $26 \mathrm{~cm}$ ureteral stents.

\begin{tabular}{|c|c|c|c|c|c|c|}
\hline & \multirow{2}{*}{$\begin{array}{c}\text { Group } 1 \\
\text { (Not crossing } \\
\text { midline) }\end{array}$} & \multirow{2}{*}{$\begin{array}{l}\text { Group } 2 \\
\text { (Crossing } \\
\text { midline) }\end{array}$} & \multirow{2}{*}{$\begin{array}{c}\text { Univariate } \\
\text { analysisa }\end{array}$} & \multicolumn{3}{|c|}{ Multivariate analysisb } \\
\hline & & & & $p$-value & $\mathrm{OR}$ & $95 \% \mathrm{Cl}$ \\
\hline \multicolumn{7}{|l|}{ Gender } \\
\hline male & $42(91.3)$ & $43(97.7)$ & 0.18 & & & \\
\hline female & $4(8.7)$ & $1(2.3)$ & & & & \\
\hline Height (m) & $1.66(1.47-1.80)$ & $1.64(1.45-1.85)$ & 0.24 & & & \\
\hline BMI $\left(\mathrm{kg} / \mathrm{m}^{2}\right)$ & $23.5(17.8-36.1)$ & $24.1(19.7-35.2)$ & 0.40 & & & \\
\hline \multicolumn{7}{|l|}{ Stone side } \\
\hline left & $29(63.0)$ & $29(65.9)$ & 0.78 & & & \\
\hline right & $17(37.0)$ & $15(34.1)$ & & & & \\
\hline C-P & $30(26.0-34.8)$ & $28.2(24.6-29.9)$ & $<0.001$ & $<0.001$ & 3.003 & $1.701-5.301$ \\
\hline P-V & $21.0(17.7-27.1)$ & $19.5(17.4-22.3)$ & $<0.001$ & 0.273 & 1.018 & $0.986-1.051$ \\
\hline
\end{tabular}

aMann-Whitney U-test; bLogistic regression analysis; $\mathbf{O R}=$ odds ratio; $\mathbf{C l}$ = confidence interval 
Figure-4 shows the comparison of the ROC curves of C-P and P-V and the AUROC in the patients with $24-$ and $26-\mathrm{cm}$ ureteral stents. The cutoff points of the ROC curve of C-P and P-V in the patients with 24-cm ureteral stents were 27.1 and $19.6 \mathrm{~cm}$, respectively, and those in the patients with 26-cm stents were 29.4 and $20.5 \mathrm{~cm}$, respectively. Comparison of the ROC curves of C-P and $\mathrm{P}-\mathrm{V}$ showed that $\mathrm{C}-\mathrm{P}$ was superior to $\mathrm{P}-\mathrm{V}$ in both the 24- and 26-cm groups ( $<<0.01)$.

\section{DISCUSSION}

Determination of the appropriate ureteral stent length is very important for reducing stent-related complications. However, few reports have addressed this topic, and no guidelines regarding ureteral stents exist. In this study, we evaluated several patient parameters that we considered relevant when choosing the appropriate stent length. We found that the method using KUB films was more useful and simpler than the methods using patient height or CT for determining the appro- priate stent length. We measured the C-P length using KUB films. In this study, we found that a 26$\mathrm{cm}$ ureteral stent is appropriate for patients with a C-P of $\geq 29.4 \mathrm{~cm}$, that a $24-\mathrm{cm}$ ureteral stent is appropriate for patients with a C-P of 27.1 to < $29.4 \mathrm{~cm}$, and that a $22-\mathrm{cm}$ ureteral stent may be appropriate for patients with a C-P of $<27.1 \mathrm{~cm}$. The appropriate ureteral stent length was short if the C-P length was shorter. Because we considered that a short C-P or P-V means that the ureter length is also expected to be short, the appropriate ureteral stent length was also short.

Some studies have reported that crossing of a ureteral stent over the bladder midline may lead to worsening of urinary symptoms $(3,10,11)$. Rane et al. (3) investigated the correlation between the position of the ureteral stent and stent-related symptoms in 60 patients and reported that a ureteral stent that crosses the bladder midline causes significantly more frequency and urgency. Ho et al. (10) evaluated whether the ureteral stent length affects stent-related symptoms after placement of stents in 87 patients. They discovered that the

Figure $4(A, B)$ - Receiver operating characteristic curves for success of ureteral stenting of KUB and CT, and area under the receiver operating characteristic curve (AUROC).

A

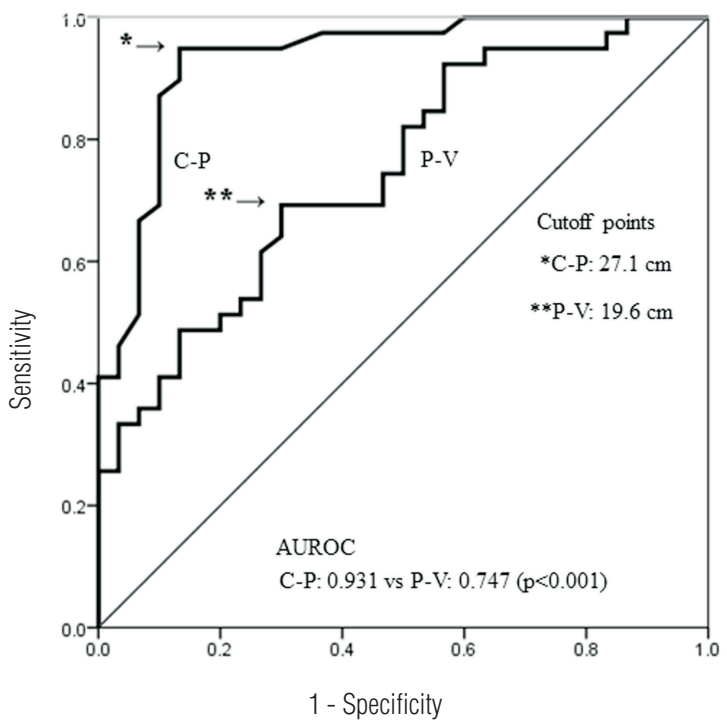

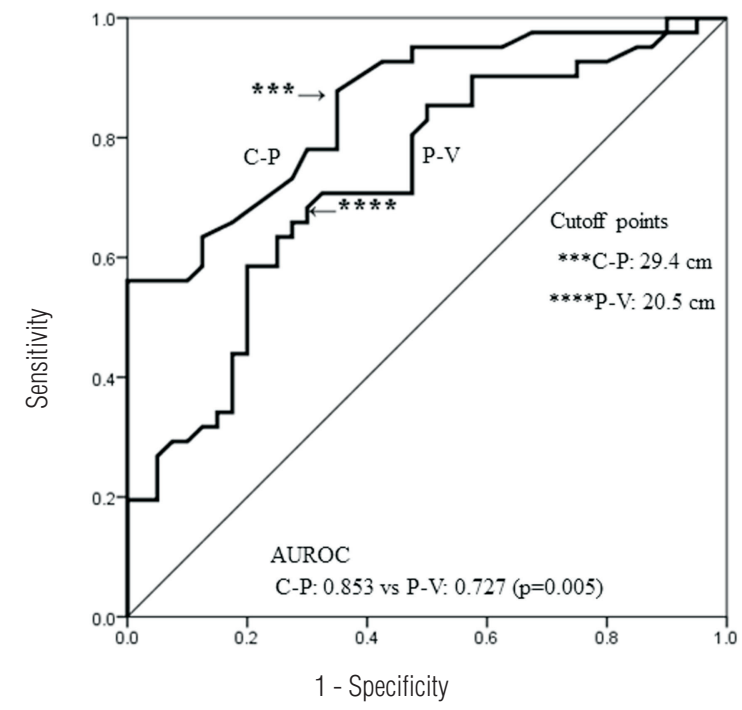

A - 24-cm ureteral stents.

B - 26-cm ureteral stents. 
ureteral stent length was associated with the position of the distal loop of the stent and reported that a longer stent crossing the bladder midline causes more irritative symptoms. Giannarini et al. (11) assessed the predictors of morbidity in 84 patients with indwelling ureteral stents. Using multivariate analyses, they reported that the location of the distal loop of the ureteral stent (not crossing the bladder midline) had the strongest association with ureteral stent-related symptoms. Therefore, we consider that crossing of a ureteral stent over the bladder midline may lead to worsening of urinary symptoms and that choosing the most appropriate ureteral stent length for each patient is important to improve stent-related symptoms.

Table- 3 shows reported clinical studies to choose the appropriate ureteral stent length not crossing the bladder midline. Pilcher and Patel (13) reported that the patient's height is a more reliable guide to choosing the most appropriate ureteral stent length than is direct ureteral measurement using a guide wire and ureteral catheter. They compared the accuracy of a patient height-based formula for choosing the correct ureteral stent length with that of direct ureteral length measurement. In their study, the patient's height correctly predicted the appropriate stent length in the majority of ureters, and direct ureteral measurement oversized the ureteral stent length in 83\% of cases (13). Additionally, Ho et al. (20) found a $22-\mathrm{cm}$ ureteral stent to be more appropriate for patients of $<175 \mathrm{~cm}$ in height, who comprised nearly $90 \%$ of their study population. Lee et al. (21) also reported that a $22-\mathrm{cm}$ stent was appropriate for patients of $<175 \mathrm{~cm}$ in height. Conversely, Jeon et al. (16) found direct measurement of the ureteral length to be a more reliable method than determination of the stent length according to patient height. Wills et al. (17) reported that measurement of the ureteral length by intravenous

Table 3 - Clinical studies performed to choose the appropriate ureteral stent length that does not cross the bladder midline.

\begin{tabular}{|c|c|c|c|}
\hline Study & $\mathrm{n}$ & Methods to choose stents & Outcome \\
\hline Pilcher and Patel (13) & 41 & Ureteral catheter vs patient's height & Patient's height was a more reliable guide. \\
\hline Ho et al. (20) & 408 & Comparing patient's height and stent position & $\begin{array}{l}\text { Patient's height could predict the ideal stent } \\
\text { length. }\end{array}$ \\
\hline Lee et al. (21) & 70 & Comparing patient's height and stent position & $\begin{array}{c}\text { A } 22 \mathrm{~cm} \text { ureteral stent was appropriate for } \\
\text { Korean patients smaller than } 175 \mathrm{~cm} \text { in } \\
\text { height. }\end{array}$ \\
\hline Jeon et al. (16) & 70 & $\begin{array}{l}\text { Direct measurement using guidewire vs } \\
\text { patient's height }\end{array}$ & $\begin{array}{c}\text { Direct measurement of ureteral length using } \\
\text { guidewire was easy and reliable. Patient's } \\
\text { height did not correlate well with appropriate } \\
\text { ureteral length. }\end{array}$ \\
\hline Wills et al. (17) & 40 & $\begin{array}{l}\text { Comparing with the ideal stent length and the } \\
\text { length of the ureter measured on intravenous } \\
\text { urography }\end{array}$ & $\begin{array}{l}\text { Measuring on intravenous urography had } \\
\text { the correlation with the ideal stent length. }\end{array}$ \\
\hline Barrett et al. (22) & 59 & $\begin{array}{l}\text { Patient's height vs L1-L5 height vs length } \\
\text { measured on CT }\end{array}$ & $\begin{array}{l}\text { CT measurements could be used to choose } \\
\text { the appropriate stent length. }\end{array}$ \\
\hline Our study & 168 & $\begin{array}{c}\text { Comparing predictors (sex, patient's } \\
\text { height, BMI, side, KUB radiograph, CT) to } \\
\text { determinate the appropriate length of ureteral } \\
\text { stent. }\end{array}$ & $\begin{array}{l}\text { KUB radiograph and CT were significant } \\
\text { factor affecting the position of the ureteral } \\
\text { stents according to our multivariate } \\
\text { analysis. }\end{array}$ \\
\hline
\end{tabular}


urography is useful. However, this method requires a full-length intravenous urography film, and tracing the curved ureter viewed on a retrograde or intravenous pyelography film is difficult (21). Therefore, we considered that establishment of a simple method with which to determine the appropriate ureteral stent length was necessary and recommend the herein-described method using KUB films, which we consider more useful and simpler than other methods.

Barrett et al. (22) reported using CT to choose the most appropriate stent length; in this technique, the ureteral length can be measured by identifying the location of the ureter in each CT slice. We referred to this method to measure the index using CT in this study. However, this method requires considerable time and effort. Moreover, CT has some limitations such as radiation exposure, measurement error associated with slice thickness, and the need for precise measurement using rendering software. Furthermore, the location of the ureteral orifices differs according to whether bladder filling is performed, (23) although bladder filling was not a standard of care in the present study. Therefore, we consider that we should investigate a more useful method than CT to choose the appropriate ureteral stent length.

This report is the first to calculate cutoff points for determination of the appropriate length of ureteral stents. We have herein introduced our method using KUB films, which is inexpensive and less invasive.

This study has some limitations. First, it was a retrospective and non-randomized trial, and the choice of the ureteral stent was entirely dependent upon the operator. Second, we did not standardize the type of ureteral stents, and the coiling patterns varied among the stents. Third, we did not use 22- and 28-cm ureteral stents and thus did not evaluate the appropriate C-P length for stents of these lengths. Fourth, we did not evaluate the patient's ureteral stent-related symptoms. Future studies should involve reassessment using a $22-\mathrm{cm}$ ureteral stent and evaluation of ureteral stent-related symptoms. Fifth, the method of measurement of the index using CT did not use the coronal plane, and the method using the Pythagorean theorem might be complicated. If we use other methods when measuring the index using CT, there would be a possibility that CT is superior to KUB. Therefore, it is controversial whether these parameters could be transposed to tomography. Finally, the renal shadow was occasionally unclear because of bowel gas. Therefore, some preoperative KUB films were seldom needed. In this study, we could measure the index of all patients using KUB films because we obtained some KUB films as a preoperative assessment, and only one or two films were needed to measure the index in most cases. Furthermore, all KUB films were reviewed by a single urologist and we have not confirmed whether other urologists can measure the index using KUB. We do not consider these methods to be complicated. However, future studies should involve reassessment in multiple centers.

\section{CONCLUSIONS}

We consider that our method using KUB radiographs is useful and simple to determine the appropriate ureteral stent length. Furthermore, we can preoperatively choose an appropriate ureteral stent length compared with direct ureteral measurement using a guide wire and ureteral catheter. However, this study has some limitations and we could not conclude that the method of measurement of the index using KUB is superior to CT.

\section{ABBREVIATIONS}

KUB = kidney, ureter, bladder X-ray

ROC $=$ receiver operating characteristic

$\mathrm{QOL}=$ quality of life

PUJ = pelviureteric junction

VUJ $=$ vesicoureteric junction

$\mathrm{BMI}=$ body mass index

$\mathrm{CT}=$ computed tomography

AUROC $=$ area under the receiver operating characteristic curve

$\mathrm{CI}=$ confidence interval

\section{CONFLICT OF INTEREST}

None declared. 


\section{REFERENCES}

1. Zimskind PD, Fetter TR, Wilkerson JL. Clinical use of longterm indwelling silicone rubber ureteral splints inserted cystoscopically. J Urol. 1967;97:840-4.

2. Joshi HB, Okeke A, Newns N, Keeley FX Jr, Timoney AG. Characterization of urinary symptoms in patients with ureteral stents. Urology. 2002;59:511-6.

3. Rane A, Saleemi A, Cahill D, Sriprasad S, Shrotri N, Tiptaft R. Have stent-related symptoms anything to do with placement technique? J Endourol. 2001;15:741-5.

4. Al-Kandari AM, Al-Shaiji TF, Shaaban H, Ibrahim HM, Elshebiny YH, Shokeir AA. Effects of proximal and distal ends of double-J ureteral stent position on postprocedural symptoms and quality of life: a randomized clinical trial. J Endourol. 2007;21:698-702.

5. Damiano R, Autorino R, De Sio M, Cantiello F, Quarto G, Perdonà $S$, et al. Does the size of ureteral stent impact urinary symptoms and quality of life? A prospective randomized study. Eur Urol. 2005;48:673-8.

6. Erturk E, Sessions A, Joseph JV. Impact of ureteral stent diameter on symptoms and tolerability. $J$ Endourol. 2003;17:59-62.

7. Candela JV, Bellman GC. Ureteral stents: impact of diameter and composition on patient symptoms. J Endourol. 1997;11:45-7.

8. Lennon GM, Thornhill JA, Sweeney PA, Grainger R, McDermott TE, Butler MR. 'Firm' versus 'soft' double pigtail ureteric stents: a randomised blind comparative trial. Eur Urol. 1995;28:1-5.

9. Liatsikos EN, Gershbaum D, Kapoor R, Fogarty J, Dinlenc $\mathrm{CZ}$, Bernardo NO, et al. Comparison of symptoms related to positioning of double-pigtail stent in upper pole versus renal pelvis. J Endourol. 2001;15:299-302.

10. Ho CH, Chen SC, Chung SD, Lee YJ, Chen J, Yu HJ, et al. Determining the appropriate length of a double-pigtail ureteral stent by both stent configurations and related symptoms. J Endourol. 2008:22:1427-31.

11. Giannarini G, Keeley FX Jr, Valent F, Manassero F, Mogorovich A, Autorino $R$, et al. Predictors of morbidity in patients with indwelling ureteric stents: results of a prospective study using the validated Ureteric Stent Symptoms Questionnaire. BJU Int. 2011;107:648-54.
12. Hruby GW, Ames CD, Yan Y, Monga M, Landman J. Correlation of ureteric length with anthropometric variables of surface body habitus. BJU Int. 2007;99:1119-22.

13. Pilcher JM, Patel U. Choosing the correct length of ureteric stent: a formula based on the patient's height compared with direct ureteric measurement. Clin Radiol. 2002;57:59-62.

14. Mitty HA, Train JS, Dan SJ. Placement of ureteral stents by antegrade and retrograde techniques. Radiol Clin North Am. 1986;24:587-600.

15. Shah J, Kulkarni RP. Height does not predict ureteric length. Clin Radiol. 2005;60:812-4.

16. Jeon SS, Choi YS, Hong JH. Determination of ideal stent length for endourologic surgery. J Endourol. 2007;21:906-10.

17. Wills MI, Gilbert HW, Chadwick DJ, Harrison SC. Which ureteric stent length? Br J Urol. 1991;68:440.

18. Paick SH, Park HK, Byun SS, Oh SJ, Kim HH. Direct ureteric length measurement from intravenous pyelography: does height represent ureteric length? Urol Res. 2005;33:199-202.

19. Breau RH, Norman RW. Optimal prevention and management of proximal ureteral stent migration and remigration. J Urol. 2001;166:890-3.

20. Ho CH, Huang KH, Chen SC, Pu YS, Liu SP, Yu HJ. Choosing the ideal length of a double-pigtail ureteral stent according to body height: study based on a Chinese population. Urol Int. 2009;83:70-4.

21. Lee BK, Paick SH, Park HK, Kim HG, Lho YS. Is a $22 \mathrm{~cm}$ Ureteric Stent Appropriate for Korean Patients Smaller than $175 \mathrm{~cm}$ in Height? Korean J Urol. 2010;51:642-6.

22. Barrett K, Foell K, Lantz A, Ordon M, Lee JY, Pace KT, et al. Best Stent Length Predicted by Simple CT Measurement Rather than Patient Height. J Endourol. 2016;30:1029-32.

23. Hwang I, Kim SO, Yu HS, Hwang EC, Jung SI, Kang TW, et al. A preliminary study of the variability in location of the ureteral orifices with bladder filling by fluoroscopic guidance: the gender difference. Int Urol Nephrol. 2013;45:639-43.

Correspondence address: Makoto Taguchi, MD Department of Urology and Andrology, Kansai Medical University, 2-5-1 Shinmachi Hirakata, Osaka 573-1010, Japan Fax: + 8172 804-2068 E-mail: taguchim@takii.kmu.ac.jp 